University of Wollongong

Research Online

Faculty of Engineering and Information

Faculty of Engineering and Information

Sciences - Papers: Part B

Sciences

2017

\title{
Effects of thermal pre-treatment and recuperative thickening on the fate of trace organic contaminants during anaerobic digestion of sewage sludge
}

\author{
Shufan Yang \\ University of Wollongong, sy527@uowmail.edu.au \\ James A. McDonald \\ University of New South Wales \\ Faisal I. Hai \\ University of Wollongong, faisal@uow.edu.au \\ William E. Price \\ University of Wollongong, wprice@uow.edu.au \\ Stuart J. Khan \\ University of New South Wales
}

See next page for additional authors

Follow this and additional works at: https://ro.uow.edu.au/eispapers1

Part of the Engineering Commons, and the Science and Technology Studies Commons 


\title{
Effects of thermal pre-treatment and recuperative thickening on the fate of trace organic contaminants during anaerobic digestion of sewage sludge
}

\author{
Abstract \\ This study examined the effects of thermal pre-treatment and recuperative thickening on anaerobic \\ digestion of sewage sludge on biogas production and removal of trace organic contaminants (TrOCs). \\ Thermal pre-treatment and recuperative thickening resulted in approximately $15 \%$ increase in biogas \\ production. However, the effects of thermal pretreatement and recuperative thickening on anaerobic \\ digestion performance in respect to the removal of TrOCs were less obvious and varied widely depending \\ on the molecular properties of each compound. Of the 40 TrOCs monitored in this study, 16 TrOCs were \\ detected in all primary sludge samples. Removal from the aqueous phase was negligible for most of \\ these 16 TrOCs. Caffeine and paracetamol were the only two TrOCs with a high removal from the \\ aqueous phase. In comparison to the aqueous phase, TrOC removal from the solid phase was \\ considerably higher. Through a mass balance calculation, it was shown that thermal pre-treatment or a \\ combination of thermal pre-treatment and recuperative thickening could enhance the biodegradation of \\ five persistent TrOCs, namely TCEP, verapamil, clozapine, triclosan, and triclocarban by $17-50 \%$.

\section{Disciplines} \\ Engineering | Science and Technology Studies

\section{Publication Details} \\ Yang, S., McDonald, J., Hai, F. I., Price, W. E., Khan, S. J. \& Nghiem, L. D. (2017). Effects of thermal pre- \\ treatment and recuperative thickening on the fate of trace organic contaminants during anaerobic \\ digestion of sewage sludge. International Biodeterioration and Biodegradation, 124 146-154.

\section{Authors} \\ Shufan Yang, James A. McDonald, Faisal I. Hai, William E. Price, Stuart J. Khan, and Long D. Nghiem
}



thickening on the fate of trace organic contaminants during anaerobic digestion of sewage sludge

\author{
Revised manuscript
}

Submitted to International Biodeterioration \& Biodegradation

6

June 2017

D. Nghiem ${ }^{a^{*}}$

9 a Strategic Water Infrastructure Lab, School of Civil, Mining and Environmental Engineering,

University of Wollongong, Australia

$11{ }^{\mathrm{b}}$ Strategic Water Infrastructure Lab, School of Chemistry, University of Wollongong

12 Australia

${ }^{c}$ School of Civil and Environmental Engineering, University of New South Wales, NSW

$16 *$ Corresponding author. Tel: 6124221 4590, Email: longn@uow.edu.au 
17 Abstract: This study examined the effects of thermal pretreatement and recuperative thickening on anaerobic digestion of sewage sludge on biogas production and removal of trace organic contaminants (TrOCs). Thermal pre-treatment and recuperative thickening resulted in approximately $15 \%$ increase in biogas production. However, the effects of thermal pretreatement and recuperative thickening on anaerobic digestion performance in respect to the removal of TrOCs were less obvious and varied widely depending on the molecular properties of each compound. In total, 16 TrOCs were detected in all primary sludge samples. Removal from the aqueous phase was negligible for most of these 16 TrOCs. Caffeine and paracetamol were the only two TrOCs with a high removal from the aqueous phase. In comparison to the aqueous phase, TrOC removal from the solid phase was considerably higher. Through a mass balance calculation, it was shown that thermal pre-treatment or a combination of thermal pre-treatment and recuperative thickening could enhance the biodegradation of five persistent TrOCs, namely TCEP, verapamil, clozapine, triclosan, and triclocarban by 17 to $50 \%$.

31 Keywords: Anaerobic digestion, thermal pre-treatment, recuperative thickening, biogas, traces organic contaminants, biodegradation. 


\section{Introduction}

Urbanization and continuous population growth have imposed an increasing demand on wastewater treatment plants (WWTPs) particularly in regard to the management of sewage sludge. In Australia, sewage sludge production (as dried solids) has increased from 0.30 to 0.33 million tonnes between 2010 to 2013 (Semblante et al., 2014). Sewage sludge contains biodegradable organics and an array of pathogens. Thus, sewage sludge treatment is necessary before any beneficial use or land disposal. Anaerobic digestion is currently the most widely used technique for sewage sludge treatment. Anaerobic digestion is a biological process in which microorganisms convert biodegradable materials in the absence of oxygen to biogas and more stable organics. It is well established that anaerobic digestion can efficiently stabilise organic materials and remove pathogenic agents in sewage sludge while simultaneously producing valuable biogas (Sawatdeenarunat et al., 2016; Sihuang et al., 2016; Tuyet et al., 2016). Biogas is a form of renewable fuel, which can be used to generate electricity and heat (Nghiem et al., 2017). The remaining and more stable solids are rich in nutrient and organics, thus, can be used for soil amendment (Nghiem et al., 2017).

Anaerobic digestion consists of four stages with hydrolysis being the first during which organic materials are transformed to fatty acids and other soluble organic compounds (Habiba et al., 2009). Since hydrolysis is the rate limiting step during anaerobic digestion, several pretreatment methods, including thermal hydrolysis, biological treatment, ultrasonication, and ozonation, have been suggested to increase the digestion rate or improve the inherent degradability of sewage sludge (Carrère et al., 2010; Dhar et al., 2012). Thermal hydrolysis is a promising pre-treatment method to improve methane production during anaerobic processing (Supplementary data Table S1) since complex organic molecules can be transformed into short-chain fragments better suited for biological digestion (Liao et al., 2016; Mottet et al., 2009; Schieder et al., 2000). The effects of thermal pre-treatment at 
temperature of up to $180{ }^{\circ} \mathrm{C}$ and duration up to 2 hours on anaerobic digestion performance have been evaluated by several recent studies (Bougrier et al., 2008; Kim et al., 2003; PérezElvira and Fdz-Polanco, 2012; Phothilangka et al., 2008; Valo et al., 2004). The optimal temperature of thermal hydrolysis was reported to be $150-180{ }^{\circ} \mathrm{C}$ by Bougrier et al. (2008) for a pre-treatment duration of 30 to 60 minutes. Thermal hydrolysis has been successfully used at a full scale wastewater treatment plant (Kepp et al., 2000). The energy balance calculation showed the net electricity production due to enhanced biogas production increased by over $20 \%$, which is more than the energy input for thermal hydrolysis.

In addition to thermal pre-treatment, recuperative thickening has also been identified as a potentially cost-effective and readily implementable method to improve anaerobic digester performance without the need to increase the size of the digester (Cobbledick et al., 2016). Recuperative thickening can increase the solids retention time (SRT) independently of the hydraulic retention time (HRT) by thickening a proportion of the digestate to remove water and then returning the thickened sludge back to the digester (Reynolds et al., 2001; Torpey and Melbinger, 1967; Yang et al., 2015). The increase in SRT helps to improve the conversion of organics to methane and increase the volatile solid (VS) reduction (Sieger et al., 2004; Yang et al., 2015). Recuperative thickening has been successfully applied in a few WWTPs in North America and Australia. Full scale monitoring data suggest that recuperative thickening can improve both biogas production and VS reduction by 15-30\% (Greer, 2011; Reynolds et al., 2001).

A major issue associated with beneficial reuse of reclaimed water and biosolids from sewage treatment is the ubiquitous occurrence of trace organic contaminants (TrOCs) in municipal wastewater. These TrOCs include several groups of widely used compounds including pharmaceuticals and personal care products, steroid hormones, industrial chemicals, pesticides, phytoestrogens, and UV filters. Their toxicological effects on human and other 
biota even at a very low concentration (less than $1 \mu \mathrm{g} / \mathrm{L}$ ) remain largely unknown but are generally suspected (Luo et al., 2014). Some TrOCs can partition from the aqueous phase in wastewater to the solid phase in sludge during wastewater treatment (Citulski and Farahbakhsh, 2010; Semblante et al., 2015). When applied to farm land, these TrOCs may accumulate in soil, presenting a potential risk to human health and the ecosystem (Citulski and Farahbakhsh, 2010). However, to date, there have been only a few investigations on the removal of TrOCs from sewage sludge by anaerobic treatment.

Of a particular note, little is known about the impact of pre-treatment on the removal of TrOCs from sewage sludge by anaerobic digestion. In a systematic lab-scale study, McNamara et al. (2012) observed no discernible impact of thermal hydrolysis on the degradation of nonylphenol ethoxylates by anaerobic digestion. Similarly, Carballa et al. (2006) reported that thermal pre-treatment of sewage sludge had no observable impact on the removal of several pharmaceuticals, musks, and steroid hormones. By contrast, Hamid and Eskicioglu (2013) observed a notable increase in the removal of estrone and estradiol by anaerobic treatment following microwave-assisted pre-treatment (80 to $160{ }^{\circ} \mathrm{C}, 2.45 \mathrm{GHz}$, $1200 \mathrm{~W}$ ). Given the paucity of information on this important issue, the present study aims to evaluate the influence of thermal hydrolysis and recuperative thickening on the fate of TrOCs in sewage sludge during anaerobic digestion. The influence of thermal hydrolysis and recuperative thickening on anaerobic digestion performance in terms of biogas production and organics removal is also investigated.

\section{Materials and Methods}

\subsection{Lab-scale anaerobic digester and sludge}

Three lab-scale anaerobic digesters previously described by Yang et al. (2016) were used in this study (Fig 1). Briefly, each digester consisted of a 28 L stainless steel reactor (Core 
Brewing Concepts, Victoria, Australia), a peristaltic hose pump (DULCO®flex from

ProMinent Fluid Controls, Australia), a temperature control unit (Neslab RTE 7), a thermal couple with temperature gauge, a biogas counter, and a gas trap for biogas sampling. One

111 digester (denoted as D1) was operated as the control system without thermal pre-treatment 112 and recuperative thickening. One digester (denoted as D2) was operated with thermal pretreatment. The last reactor (denoted as D3) was operated with both thermal pre-treatment and recuperative thickening. All three reactors were operated in parallel and were each seeded with $20 \mathrm{~L}$ anaerobically digested sludge. The digested sludge and primary sludge were all sampled from a full scale wastewater treatment plant in New South Wales, Australia, with average total solid (TS) content of $29.0 \pm 1.0 \mathrm{~g} / \mathrm{L}$ and $22.2 \pm 2.2 \mathrm{~g} / \mathrm{L}$, respectively. All sludge samples were stored at $4{ }^{\circ} \mathrm{C}$ until use or else discarded within two weeks.

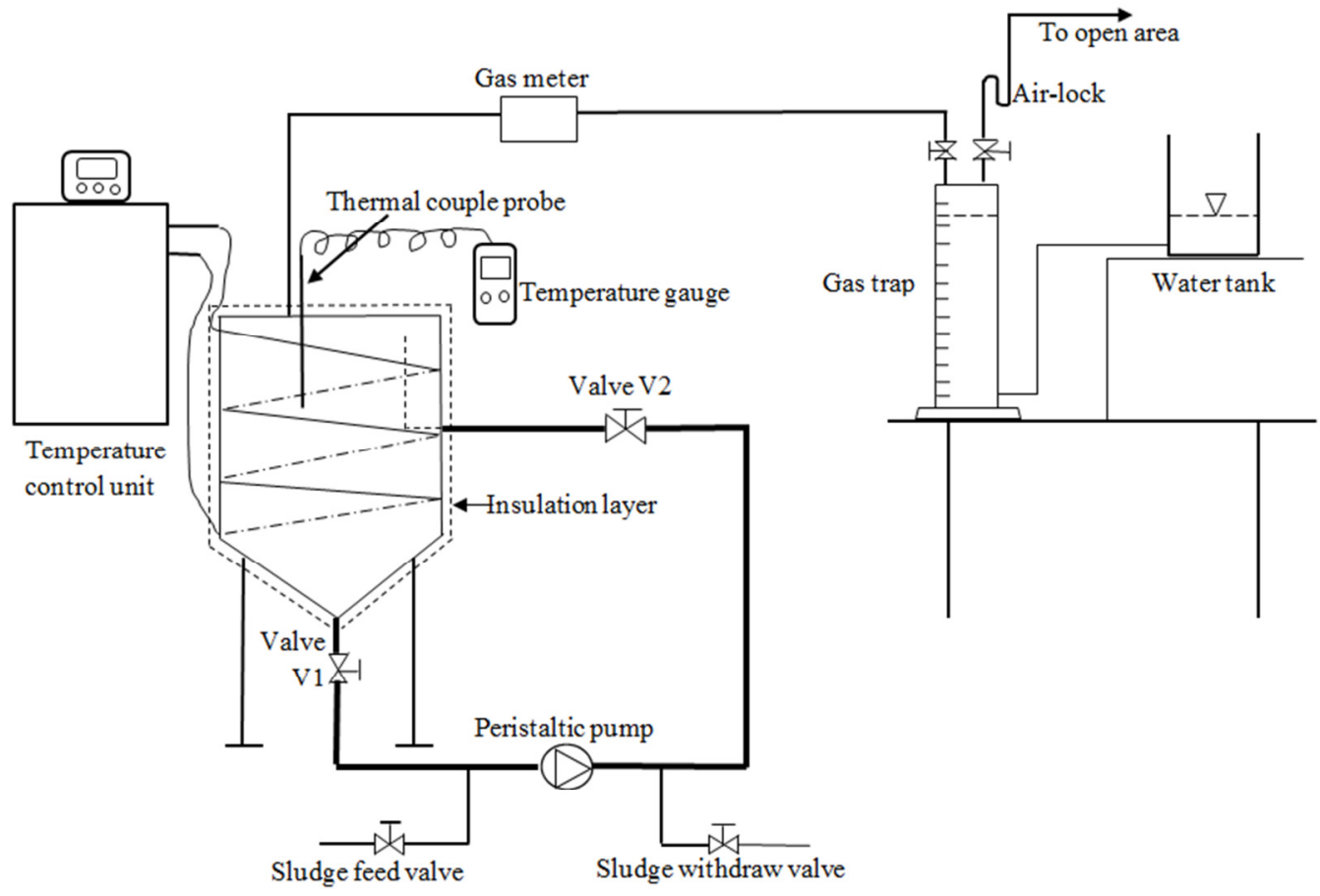

Fig 1: The schematic diagram of the three lab-scale anaerobic digesters.

121 All anaerobic digesters were operated under the same HRT of $20 \mathrm{~d}$ by wasting $1 \mathrm{~L}$ of 122 digestate and the feeding with $1 \mathrm{~L}$ of primary sludge each day. Raw sludge, thermally 
pretreated sludge, and digested sludge were collected weekly for characterisation. Parameters that were regularly measured include TS, volatile solid (VS), chemical oxygen demand (COD), alkalinity and $\mathrm{pH}$.

\subsection{Thermal pre-treatment}

The feed sludge to digester D2 and D3 was thermally pretreated at $150{ }^{\circ} \mathrm{C}$ and $500 \mathrm{kPa}$ for 30 minutes using a New Tek Machinery pressure vessel (Changzhou, China) with a heating jacket. At the conclusion of the process, the pressure inside the vessel was released and the sludge was allowed to cool to room temperature (ca. $25^{\circ} \mathrm{C}$ ) before feeding to the digester.

\subsection{Recuperative thickening}

Digester D3 was operated with recuperative thickening to achieve an SRT of $30 \mathrm{~d}$ with the HRT at 20 d i.e., same as the other digesters. A thickening ratio of 1.33 (which is the ratio of the total TS from primary sludge feed and return thickened sludge over the TS from primary sludge feed) was used. Each day, $2 \mathrm{~L}$ of the digestate was withdrawn from digester D3 and dosed with thickening polymer (Zetag $8169, \mathrm{BASF})$ at $7.5 \mathrm{~g} / \mathrm{Kg}$ dry sludge. The sludge was gently mixed and allowed to settle by gravity for at least 10 minutes. $1 \mathrm{~L}$ of thickened sludge was then mixed with the thermally pretreated $\left(150{ }^{\circ} \mathrm{C}, 30 \mathrm{~min}\right)$ primary sludge $(1 \mathrm{~L})$ to form $2 \mathrm{~L}$ of feed to return to the digester. The excess thickened sludge and supernatant were discarded.

\subsection{Analytical methods}

\subsubsection{Anaerobic digestion performance}

Biogas production rate was monitored daily by a custom-made gas counter (Yang et al., 2016). The biogas composition was detected weekly by a portable gas analyser (GA5000 gas analyser, Geotechnical Instruments Ltd, UK) (Nghiem et al., 2014). Additionally, samples 
from primary sludge (before and after thermal treatment) and digested sludge were taken 147 weekly to analyse sludge characters such as TS, VS, total COD (tCOD), soluble COD (sCOD), $\mathrm{pH}$ and alkalinity. TS, VS, and alkalinity were measured in accordance to the standard methods. COD was measured following the US-EPA Method 8000 using high range 150 plus COD vials (HACH, USA). The supernatant used for measurement of sCOD was obtained by centrifuging sludge sample at $3720 x g$ for 10 minutes (Allegra X-12R centrifuge, Beckman Coulter, Australia), and then filtering through $1 \mu \mathrm{m}$ glass microfiber filter paper (Filtech, Australia).

2.4.2 TrOC sample preparation and analysis

Primary and digested samples were collected every 7 days to prepare duplicate samples for TrOC concentration analysis (Wijekoon et al., 2015). Sludge samples were centrifuged at 3720xg for 10 minutes (Alleegra X-12R, Beckman Coulter, USA) to obtain solid pellets and supernatant for further processing. Supernatant from sludge sample $(50 \mathrm{~mL})$ was diluted with Milli-Q to $500 \mathrm{~mL}$. Then the obtained aqueous samples were filtered by $1 \mu \mathrm{m}$ and then 0.7 $\mu \mathrm{m}$ pore size glass fiber filter paper. The filtered samples were spiked with surrogate $(50 \mu \mathrm{L}$ per sample) containing 40 isotopically labelled standards for method recovery and determine TrOC concentration before proceeding to solid phase extraction (SPE). During the SPE, HLB cartridges were conditioned with $5 \mathrm{~mL}$ methyl tert-butyl ether, $5 \mathrm{~mL}$ methanol, and $2 \times 5 \mathrm{~mL}$ Milli-Q water before the liquid samples were loaded to the cartridges at the flow rate of approximately $15 \mathrm{~mL} / \mathrm{min}$. After concentrating to $1 \mathrm{~mL}$, eluted samples were subjected to high performance liquid chromatography-tandem mass spectrometry analysis (HPLC-MSMS) (Alturki et al., 2013). In this study, a spectrum of 40 TrOCs was used to prepare the surrogate and screen the TrOC concentration of sludge samples. 

The solid pellets were freeze-dried using an Alpha 1-2 LDplus Freeze dryer (Christ GmbH, Germany). The dried samples were ground to powder and $0.5 \mathrm{~g}$ was transferred to a $13 \mathrm{~mL}$ glass vial (with cap) for extraction. Methanol $(10 \mathrm{~mL})$ was added to the vial, mixed with the powder by a vortex mixer (VM1, Ratek, Australia), and ultrasonicated for 10 minutes at $40{ }^{\circ} \mathrm{C}$. The solution was then centrifuged at $3720 \mathrm{xg}$ for 10 minutes to obtain a supernatant. The residual solid was extracted using $10 \mathrm{~mL}$ solvent made of dichloromethane and methanol $(1: 1, \mathrm{v} / \mathrm{v})$ by repeating the previous steps. The supernatants from these steps were combined and diluted to $500 \mathrm{~mL}$ by Milli-Q water. The liquid samples were then filtered, spiked with surrogate, loaded to the SPE cartridges and analysed following the same procedure for sludge supernatant samples described before.

\subsubsection{TrOC mass balance}

Mass balance calculations were conducted for each $\operatorname{TrOC}$ to determine their fate in the aqueous and solid phase (Wijekoon et al., 2015). The total mass of each TrOC fed into the system can be described as:

$M_{\text {in }}=X_{i n} \times T S_{P S}+S_{\text {in }}$

where $M_{\text {in }}$ is the total mass of TrOC in $1 \mathrm{~L}$ of feed (ng), $X_{i n}$ is the TrOC concentration in the solid phase of primary sludge (ng/g dry sludge), $T S_{P S}$ is the total solid concentration of primary sludge $(\mathrm{g} / \mathrm{L})$, and $S_{\text {in }}$ is the TrOC concentration in the aqueous phase of primary sludge (ng/L). The mass of $\operatorname{TrOC}\left(\mathrm{M}_{\mathrm{aq}}\right)$ in the aqueous phase in $1 \mathrm{~L}$ of the digestate can be measured experimentally. The mass of TrOC in the solid phase of the digestate can be described as:

$M_{\text {solid }}=X_{\text {solid }} \times T S_{D S}$ 
where $M_{\text {solid }}$ is the mass of TrOC in the solid phase (ng), $X_{\text {solid }}$ is the TrOC concentration in the solid phase of digested sludge (ng/g dry sludge), $T S_{D S}$ is the total solid concentration of digested sludge (g/L). Thus the mass balance for TrOC concentration can be presented as

$$
M_{\text {in }}=M_{a q}+M_{\text {solid }}+M_{\text {bio }}
$$

where $M_{b i o}$ is the mass of TrOC that has been biodegraded.

\section{Results and discussion}

\subsection{Thermal pre-treatment and recuperative thickening}

Thermal pre-treatment and recuperative thickening (Digester D3) resulted in approximately $15 \%$ increase in biogas production in comparison to the control digester (D1) (Fig 2). The combination of thermal pre-treatment and recuperative thickening (Digester D3) did not lead to any additional increase in biogas production compared to only thermal pre-treatment (D2). According to Pilli et al., (2015), thermal pre-treatment causes the disintegration and solubilisation of some solid sludge particles, thus, enhancing the hydrolysis step and hence biogas production. Indeed, in this study, in which approximately $10 \%$ of the tCOD of primary sludge was converted to sCOD after thermal treatment. On the other hand, recuperative thickening can extend the residence time of sludge in the reactor and recapture soluble macro-organic molecules for further digestion. Biogas production-increase by up to $30 \%$ has been reported in previous laboratory scale and full scale studies (Cobbledick et al., 2016; Reynolds et al., 2001). Results from Fig 2 suggest that the benefits of thermal pre-treatment and recuperative thickening are mutually exclusive. It is also noteworthy that thermal pretreatment and recuperative thickening did not exert any observable impact on biogas composition. Throughout this study, biogas composition from all three digesters was stable with approximately $60 \% \mathrm{CH}_{4}$ and $40 \% \mathrm{CO}_{2}$. 


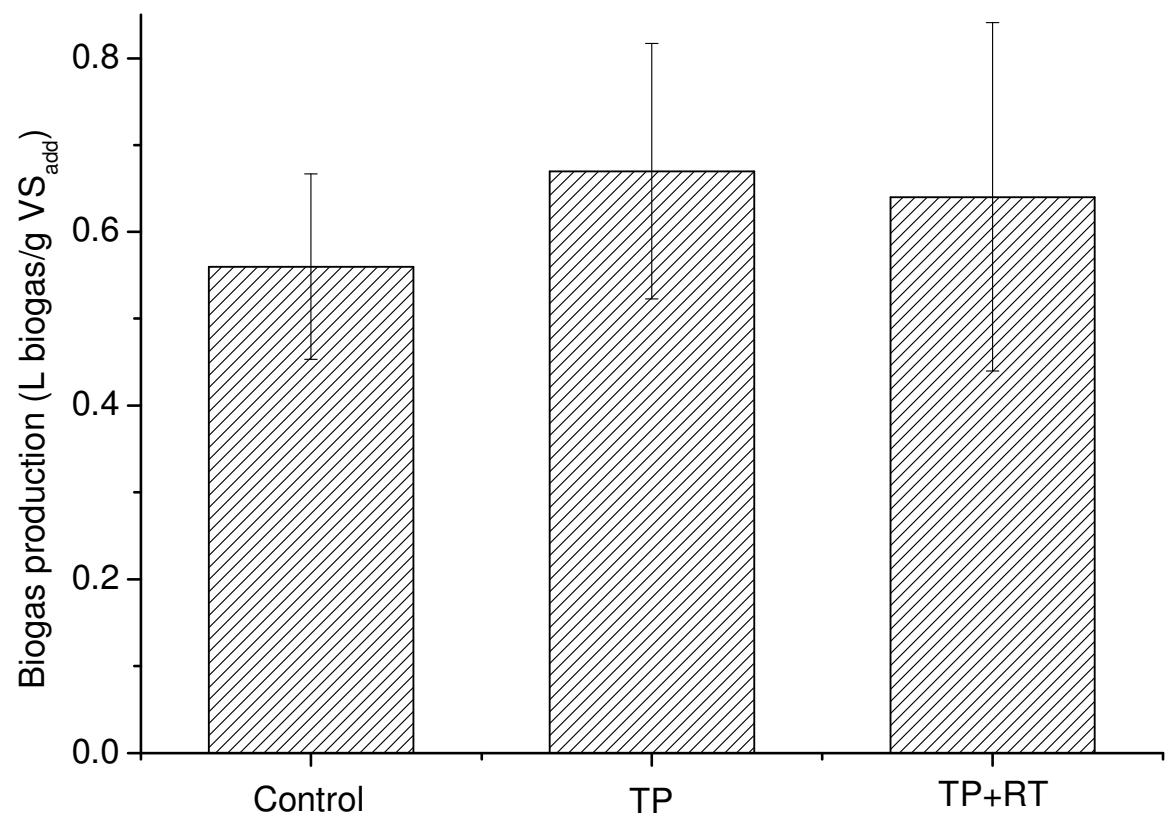

215 Fig 2: Average biogas production from digester D1 (Control), D2 (Thermal pre-treatment $216(\mathrm{TP})$ ) and D3 (Thermal pre-treatment and recuperative thickening (TP+RT)). Error bars show 217 the standard deviation of 7 measurements (one per week).

218 The sludge composition varied quite significantly throughout the course of this study. Since 219 organic removal in terms of TS, VS, tCOD and sCOD was determined on a weekly basis, 220 there were some notable variations. TS and VS removals ranged from 50 to $80 \%$ and 70 to $22190 \%$, respectively. Due to these significant variations in TS and VS, the effects of thermal 222 pre-treatment and recuperative thickening were not observable in this study. Nevertheless, 223 some enhancement in the removal of tCOD and sCOD could be observed in Fig 3 . With the 224 exception of day 49, the removal of tCOD by Digester 2 (thermal pre-treatment) and Digester 2253 (thermal pretreament and recuperative thickening) was comparable or higher than that of 226 the control digester (Fig 3a). 

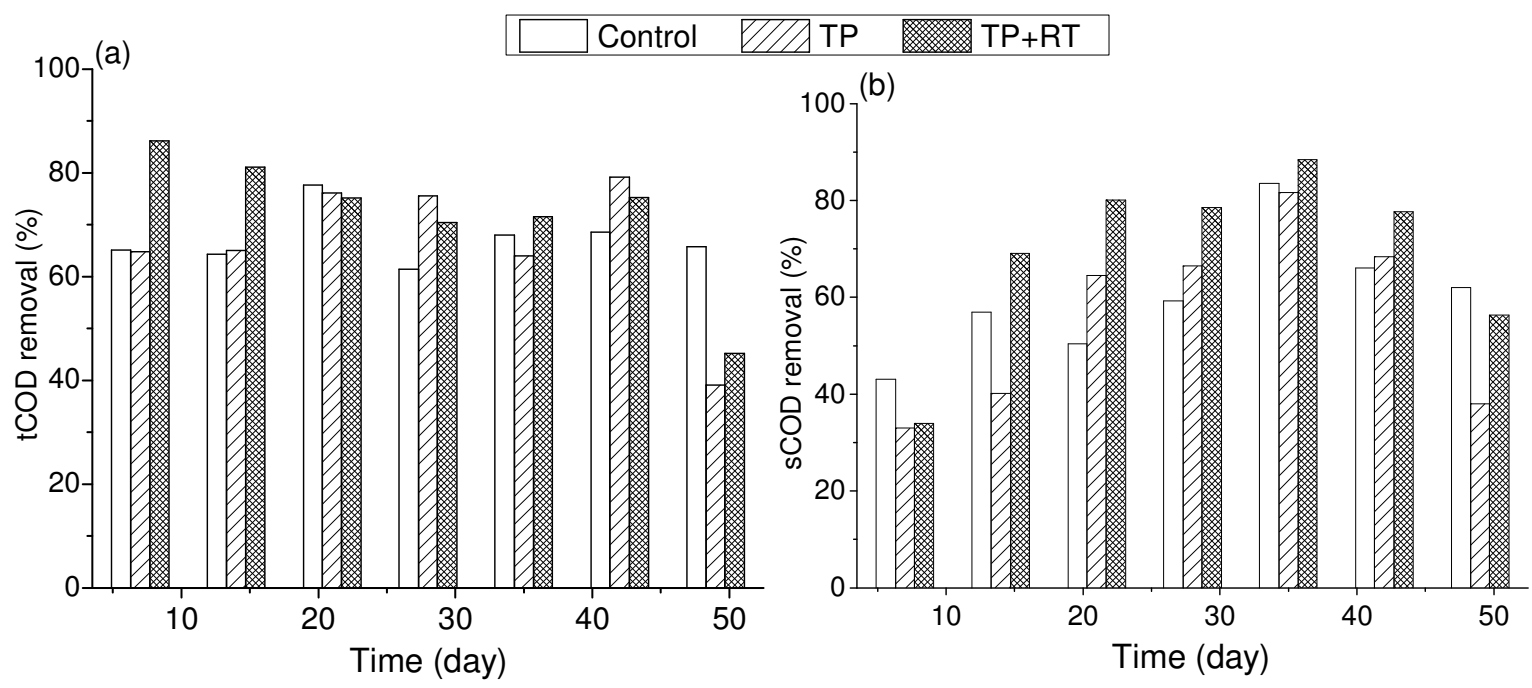

Fig 3: (a) tCOD removal and (b) sCOD removal by the control digester (D1), digester 2 with thermal pre-treatment (TP), and digester 3 with thermal pre-treatment and recuperative thickening $(\mathrm{TP}+\mathrm{RT})$.

The effect of pre-treatment and recuperative thickening on removal performance was most notable in terms of sCOD removal. Digester D2 showed comparable sCOD removal to that by the control digester (D1). On the other hand, digester D3 showed a notable increase in 234 sCOD removal (Fig 3b). As noted above, thermal pre-treatment led to the solubilisation of 235 some tCOD into sCOD. On the other hand, due to sludge thickening, soluble organics can be retained for further digestion. Thus, recuperative thickening could improve the removal of 237 sCOD.

238 Several other parameters including $\mathrm{pH}$ and alkalinity were also monitored. The mixed liquor $\mathrm{pH}$ value of all three digesters was stable between $7.0-7.5$ and the alkalinity was over 2600 mg $\mathrm{CaCO}_{3} / \mathrm{L}$ (Supplementary data Fig S4). These results confirm stable operation of all three

241 digesters in this study. 
243 In good agreement with a previous study by Yang et al. (2017), of the 40 TrOCs monitored in

244 this study, 16 compounds were prevalently detected in all primary sludge samples (Fig 4).

245 The concentrations in the aqueous and solid phase were in the range from 50 to 40,000 ng/L

246 and from 20 to nearly 9,000 ng/g dry sludge, respectively. The occurrence of these TrOCs in

247 primary sludge is well related to their usage in daily life. For examples, caffeine (which is a

248 stimulant in coffee and tea) and paracetamol (which is a widely used pain killer) were

249 detected at the highest concentration in the aqueous phase $(40,000$ and $38,000 \mathrm{ng} / \mathrm{L}$,

250 respectively). At the TS content of $29 \mathrm{~g} / \mathrm{L}$, it can also be inferred from Fig 4 that these TrOCs

251 occurred mostly in the solid phase (i.e. 70 to $100 \%$ in the total mass in primary sludge).

252 Caffeine and ibuprofen are the only two exceptions. The mass distributions of caffeine and

253 ibuprofen in the solid phase were 24 and $41 \%$, respectively, possibly because of their

254 hydrophilicity. These results highlight the need for specific investigation of the removal of

255 TrOCs from the solid phase and that data from previous studies considering only the aqueous

256 phase may not be valid in the context of anaerobic digestion. 
(a)

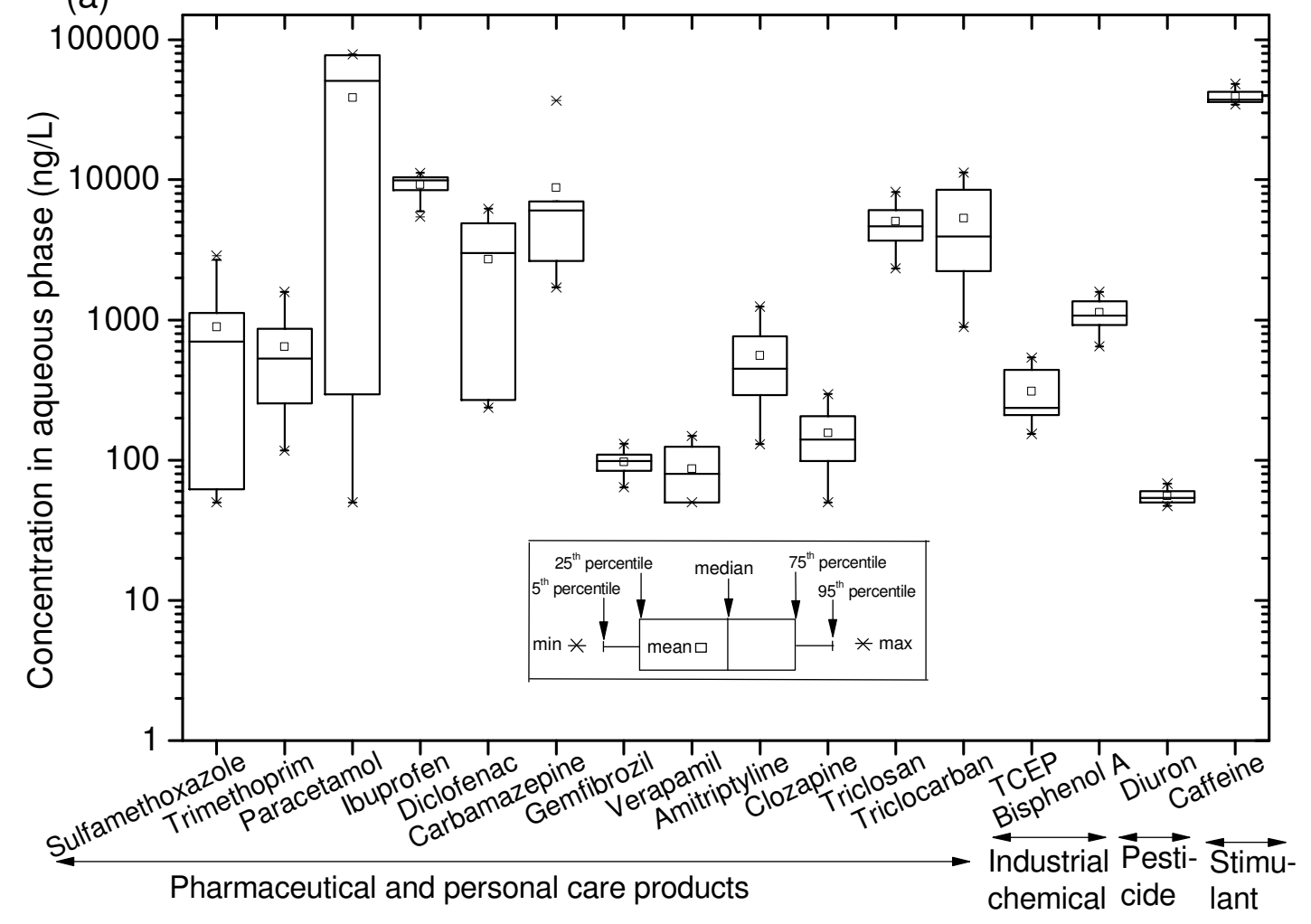

257

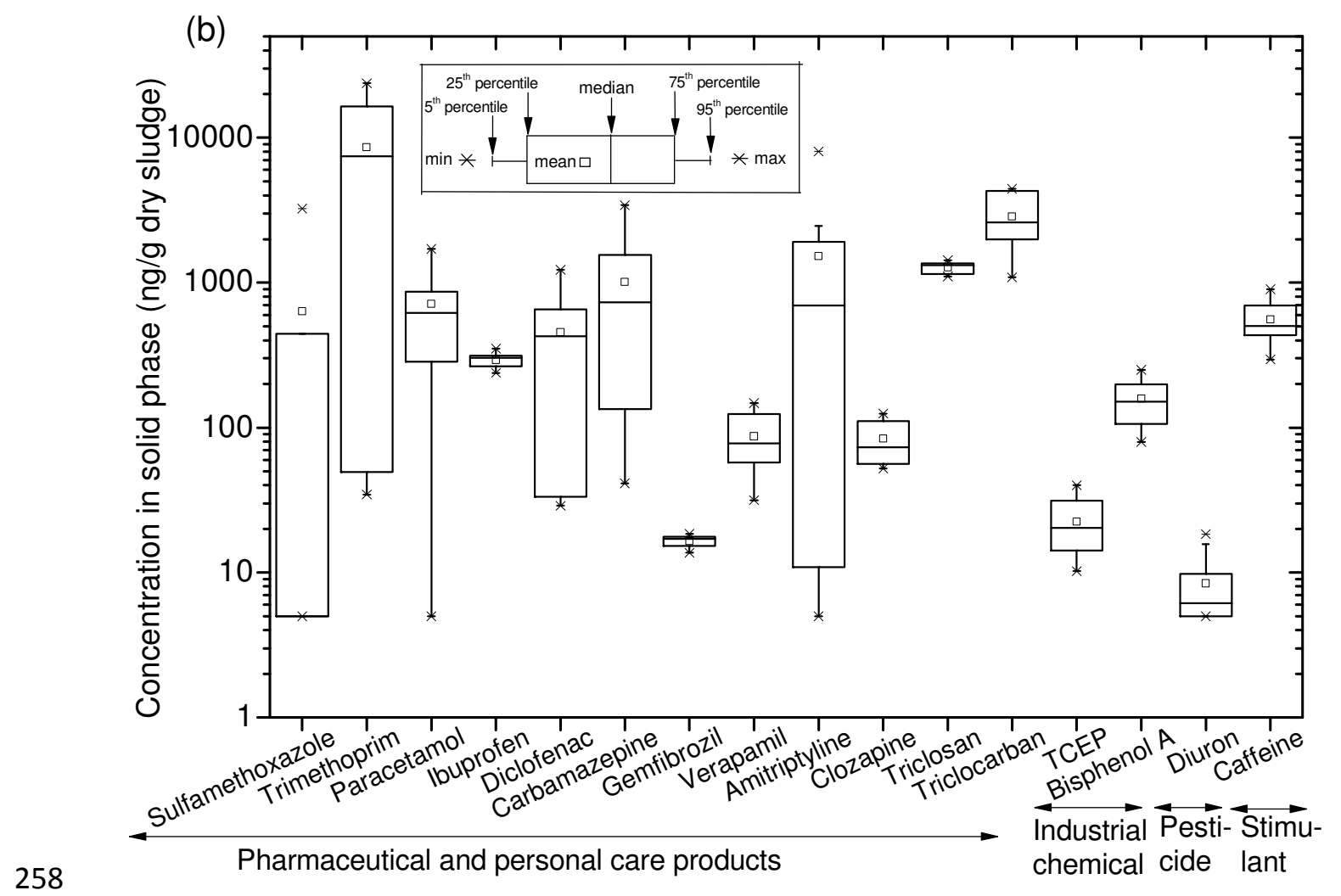

259 Fig 4: TrOC concentrations in (a) aqueous phase and (b) solid phase of primary sludge. 12 samples were taken during the experimental period. 


\subsection{TrOC removal in the aqueous and solid phase}

262 TrOC concentrations in the aqueous and solid phase of the feed and digestate from the three 263 reactors are shown in Figs. 4 and 5, respectively. In these Figs, the TrOCs were listed in the order of increasing hydrophobicity. Under all experimental conditions, caffeine and paracetamol were almost completely removed (98 - 99\%) from the aqueous phase (Fig 5). Moderate removals from the aqueous phase were observed for trimethoprim and amitriptyline, especially when pre-treatment and recuperative thickening were applied together (D3). However, all other TrOCs were not significantly removed from the aqueous phase as can be observed with all three digesters (Fig 5). In fact, in the case of ibuprofen, gemfibrozil, and diuron, their concentrations in the aqueous phase of the digestate (after anaerobic treatment) were even higher than the corresponding values of the feed primary sludge (Fig 5). It is possible that the anaerobic condition could facilitate the transfer of some TrOCs from the solid to aqueous phase. This is probably because of the transfer of TrOCs from the solid phase to the aqueous phase during anaerobic digestion. It is also noteworthy from section 3.1 that most of these TrOCs are in the solid phase.

TrOC removal from the solid phase was notably higher in comparison to that from the aqueous phase. As can be seen in Fig 6, several hydrophilic TrOCs including caffeine, sulfamethoxazole, trimethoprim and paracetamol were well removed from the solid phase by anaerobic digestion. The hydrophilicity of compounds appears to be an important factor for their high removal from solid phase since hydrophilic compounds would easily desorb from sludge granules. However, similar to the removal from aqueous phase, there is no obvious evidence that thermal pre-treatment and recuperative thickening could improve the removal of all of these TrOCs from the solid phase (Fig 6). 


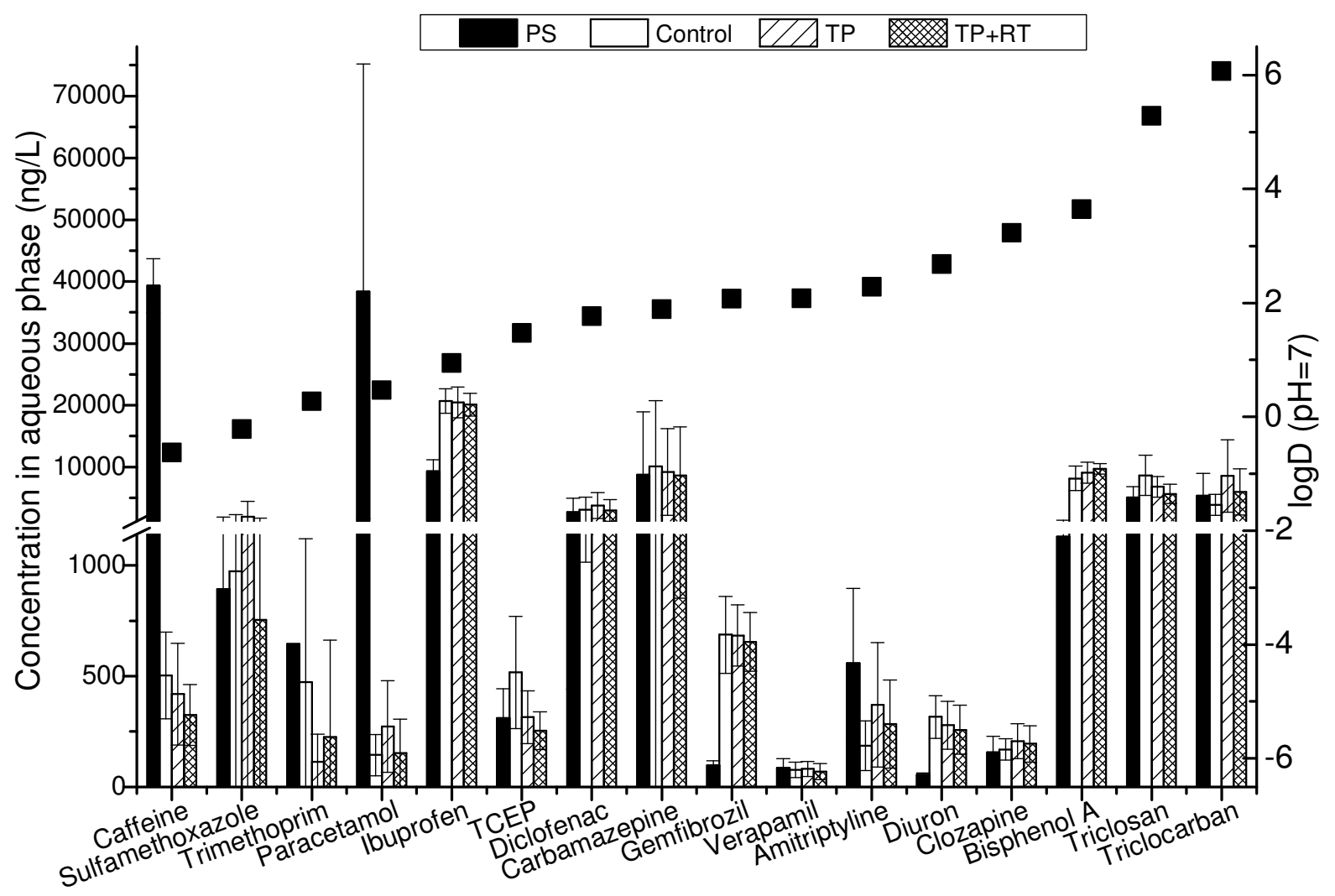

Fig 5: Average concentrations of TrOCs in aqueous phase of primary sludge (PS), digested sludge from digester D1 (Control), D2 (TP) and D3 (TP+RT) (mean \pm standard deviation of 12 samples).

Several previous studies have also shown no discernible changes in TrOC removal after thermal pre-treatment. For example, McNamara et al. (2012) reported that nonylphenol, diethoxylate and nonylphenol monoethoxylate were not removed from the influent by anaerobic treatment with and without thermal treatment $\left(150{ }^{\circ} \mathrm{C}, 2 \mathrm{~h}\right)$. Similarly, Carballa et al. (2006) also reported that thermal pre-treatment of mixed sludge by autoclaving at $130{ }^{\circ} \mathrm{C}$ for $1 \mathrm{~h}$ had no impact on the removal of various pharmaceuticals, musks, and hormones by anaerobic treatment. However, it is noteworthy that these previous studies focused on the anaerobic treatment of wastewater and only considered the aqueous phase. Thus, their results cannot readily correlate to the anaerobic digestion of wastewater sludge. As discussed above, during anaerobic digestion of sludge, the transfer of TrOCs between the aqueous and solid 

and solid phase.

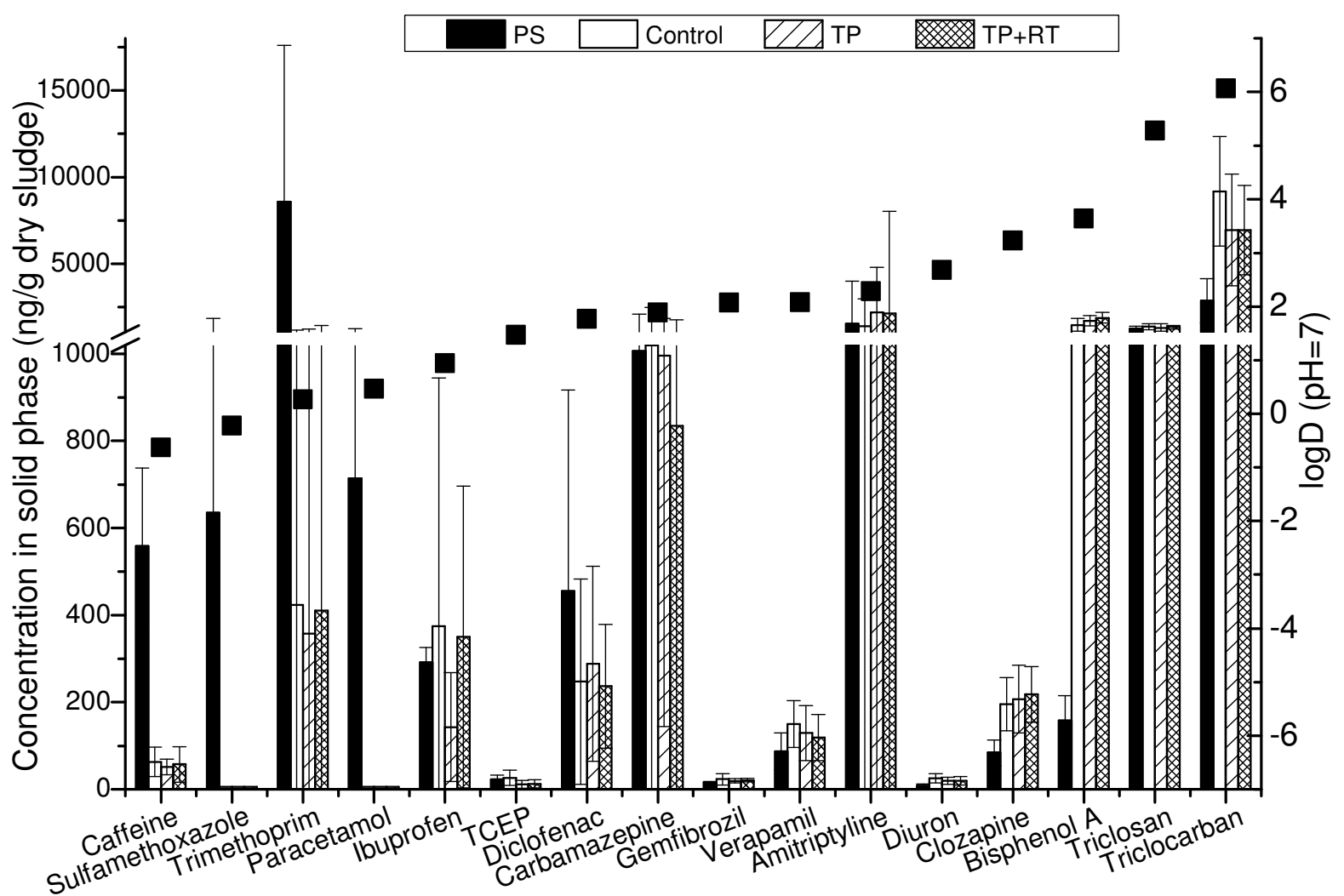

Fig 6: Average concentrations of TrOCs in solid phase of primary sludge (PS), digested sludge from digester D1 (Control), D2 (TP) and D3 (TP+RT) (mean \pm standard deviation of 12 samples).

\subsection{Fate of TrOCs during anaerobic digestion}

Fig 7 shows the fate of each TrOC amongst the three possible domain namely biodegradation, partitioning to the solid phase, and partitioning in the aqueous phase. Several readily biodegradable TrOCs can be identified from Fig 7. They include caffeine, sulfamethoxazole, trimethoprim and paracetamol (Fig 7). Likewise, four TrOCs including ibuprofen, carbamazepine, diuron and clozapine were not biodegraded under any experimental conditions in this study (Fig 7). 
313 It has been established that a compound's molecular structure is a major factor governing 314 their degradability (Tadkaew et al., 2011; Wijekoon et al., 2015; Yang et al., 2016). TrOCs with strong electron donating functional groups (Supplementary data Table S6) such as amine (caffeine, sulfamethoxazole and trimethoprim), amino (paracetamol and sulfamethoxazole), hydroxyl (paracetamol) and ether (trimethoprim) are known to be readily biodegradable. On the other hand, TrOCs with strong electron withdrawing functional groups tend to be persistent to biological treatment. Examples of these electron withdrawing functional groups are carboxyl (gemfibrozil and ibuprofen), amide group (carbamazepine), and chloro (diuron). Indeed, as can be seen in Fig 7, all TrOCs with electron withdrawing functional groups were not effectively biodegraded.

Results from this study are consistent with several previous studies. Caffeine (Narumiya et al., 2013; Yang et al., 2016), trimethoprim (Malmborg and Magnér, 2015; Narumiya et al., 2013) and sulfamethoxazole (Carballa et al., 2007; Narumiya et al., 2013) have been reported to be well removed by anaerobic digestion. By contrast, carbamazepine (Carballa et al., 2007; Malmborg and Magnér, 2015; Narumiya et al., 2013), diuron (Carballa et al., 2007; Tadkaew et al., 2011) and ibuprofen (Alvarino et al., 2014; Malmborg and Magnér, 2015) were resistant to anaerobic digestion.

Of particular note, enhanced biodegradation due to either thermal pre-treatment and/or recuperative thickening was observed with five TrOCs (denoted in Fig 7 with \#). The biodegradation of triclosan and triclocarban were improved by approximately $10 \%$ due to thermal pre-treatment (Fig 6a and b) and further improved (by about 15\%) when recuperative thickening was also applied (Fig 6c). Verapamil and clozapine were approximately 20\% more biodedegraded when both thermal pre-treatment and recuperative thickening were applied (Fig 6a and c). However, with thermal pre-treatment and recuperative thickening, TCEP biodegradation increased to approximately $40 \%$ and $60 \%$, respectively. 


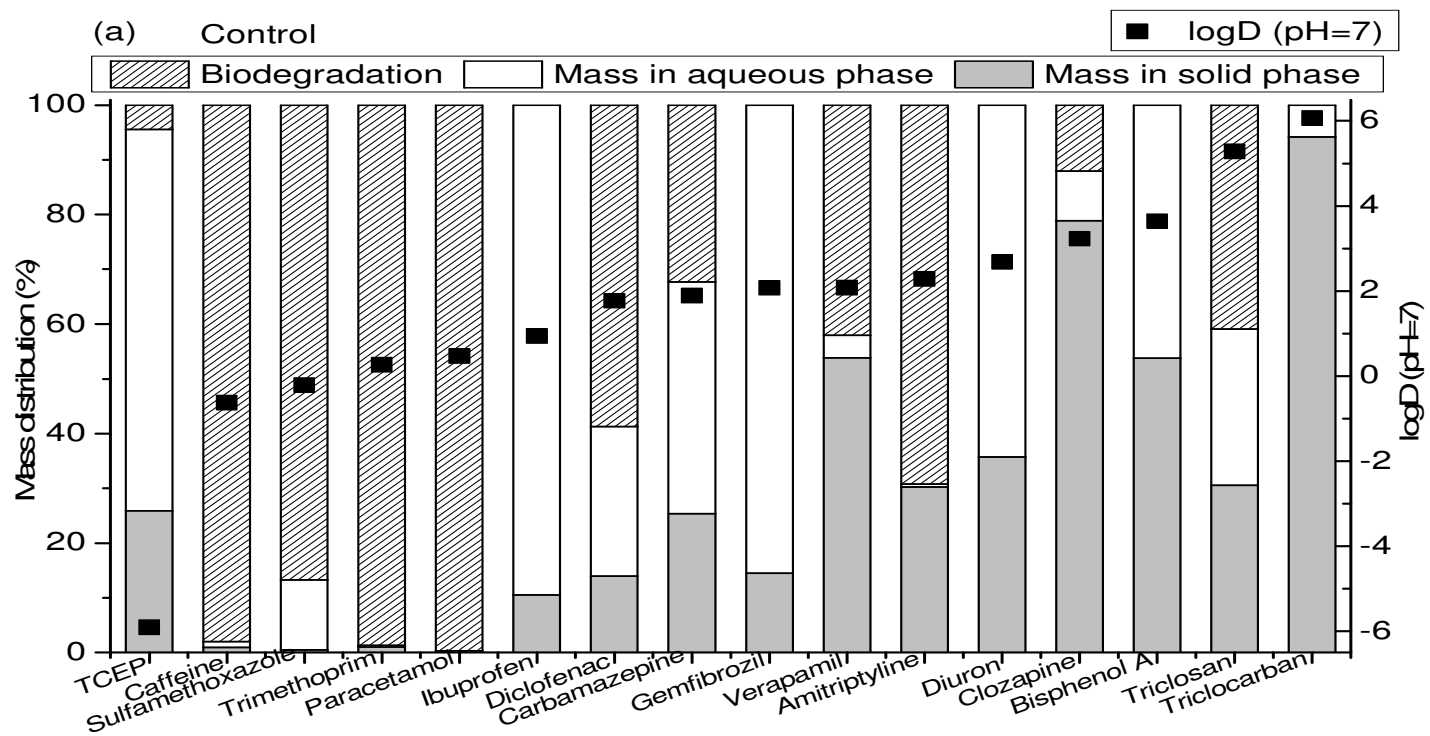

338

(b) TP

- $\log \mathrm{D}(\mathrm{pH}=7)$

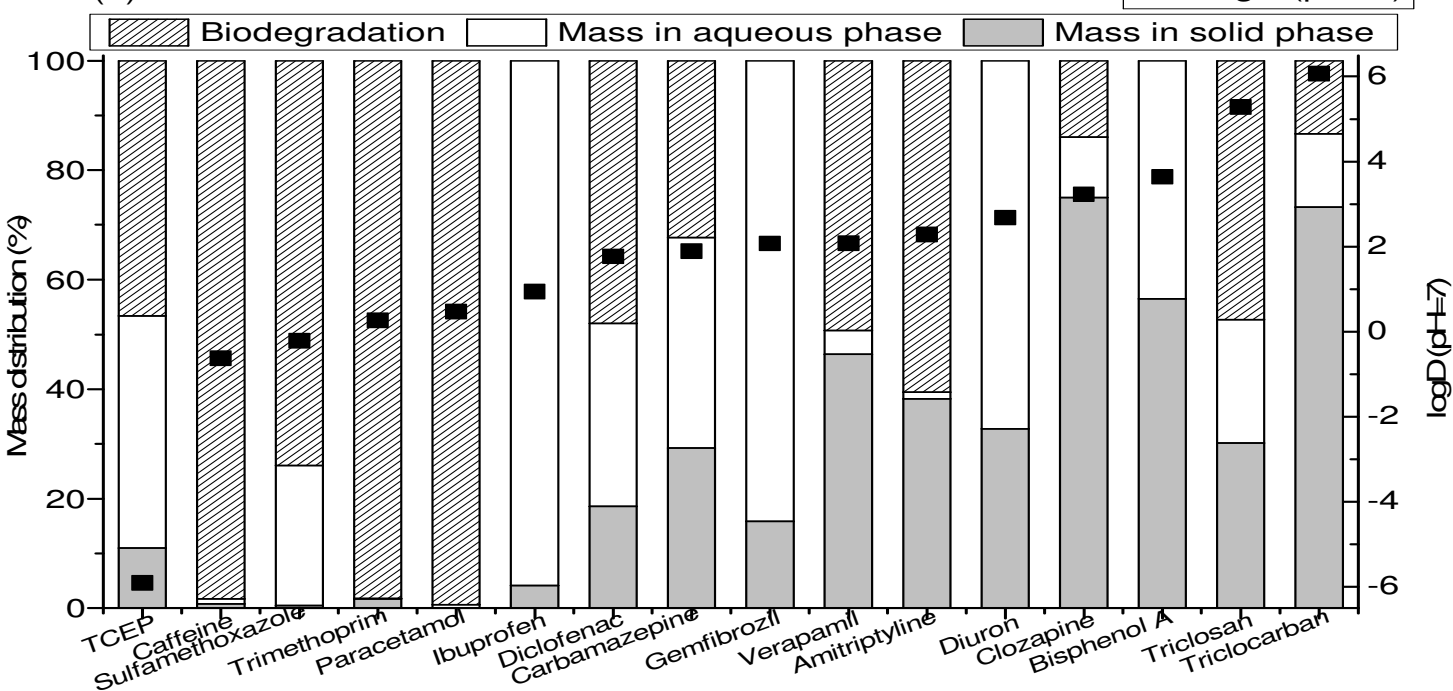

339

(c)

RT

inass in acueous io

- $\log \mathrm{D}(\mathrm{pH}=7)$

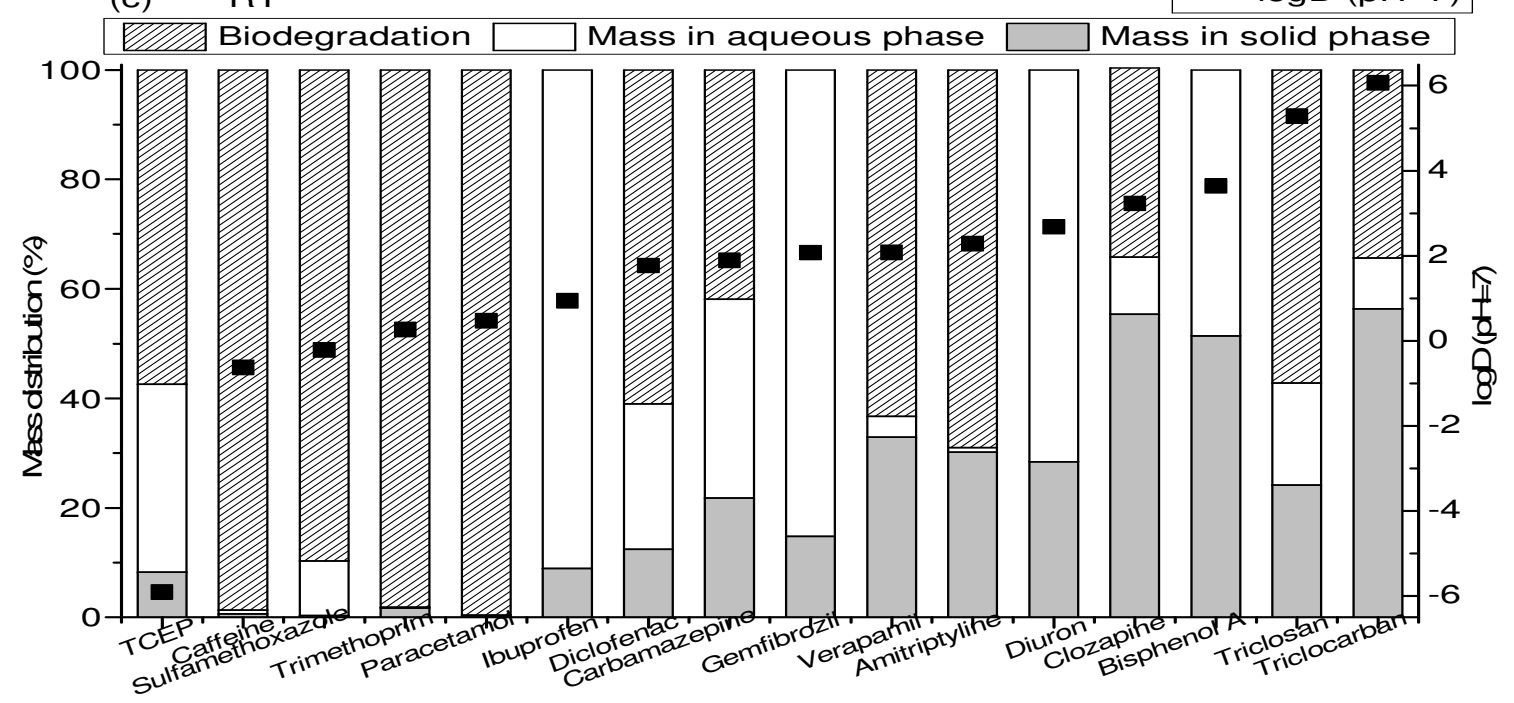

Fig 7: Overall fate of each compound by anaerobic digestion in digester (a) D1 (Control), (b) D2 (TP) and (c) D3 (TP+RT). 
344 The positive impact of thermal pre-treatment and recuperative thickening does not seem to be governed by the compound hydrophobicity. Indeed, of the 16 TrOCs in Fig 7, TCEP is highly hydrophilic while triclosan and triclocarban are the most hydrophobic. The removal of TrOCs with electron withdrawing functional groups (thus these TrOCs are inherently persistent to biodegradation) is likely to benefit from thermal pre-treatment and recuperative thickening. These TrOCs have at least one electron withdrawing functional group in their molecular structure and are known to be persistent to biodegradation.

\section{Conclusions}

352 The effects of thermal pretreatement and recuperative thickening on anaerobic digestion performance were examined in terms of biogas production and the removal of trace organic contaminants (TrOCs). Thermal pre-treatment and recuperative thickening resulted in approximately $15 \%$ increase in biogas production. In total, 16 TrOCs were detected in all primary sludge samples. The effects of thermal pretreatement and recuperative thickening on TrOC removal varied significantly. Removal from the aqueous phase was negligible for most of the 16 TrOCs detected in the primary sludge samples. Caffeine and paracetamol were the only two TrOCs with an appreciable level of removal from the aqueous phase. In comparison to the aqueous phase, TrOC removal from the solid phase was considerably higher. Through a mass balance calculation, it was shown that thermal pre-treatment or a combination of thermal pre-treatment and recuperative thickening could enhance the biodegradation of five persistent TrOCs, namely TCEP, verapamil, clozapine, triclosan, and triclocarban by 17 to $50 \% . "$ 


\section{References}

367 Alturki, A.A., McDonald, J.A., Khan, S.J., Price, W.E., Nghiem, L.D., Elimelech, M., 2013.

368 Removal of trace organic contaminants by the forward osmosis process. Separation and 369 Purification Technology 103, 258-266.

370 Alvarino, T., Suarez, S., Lema, J.M., Omil, F., 2014. Understanding the removal mechanisms

371 of PPCPs and the influence of main technological parameters in anaerobic UASB and aerobic

372 CAS reactors. Journal of Hazardous Materials 278, 506-513.

373 Bougrier, C., Delgenès, J.P., Carrère, H., 2008. Effects of thermal treatments on five different 374 waste activated sludge samples solubilisation, physical properties and anaerobic digestion.

375 Chemical Engineering Journal 139, 236-244.

376 Carballa, M., Omil, F., Lema, J.M., Alder, A.C., 2006. Comparison between the conventional anaerobic digestion of sewage sludge and its combination with a chemical or thermal pretreatment concerning the removal of pharmaceuticals and personal care products. Water

379 Science and Technology 53, 109-117.

380 Carballa, M., Omil, F., Ternes, T., Lema, J.M., 2007. Fate of pharmaceutical and personal care products (PPCPs) during anaerobic digestion of sewage sludge. Water Research 41, 2139-2150.

Carrère, H., Dumas, C., Battimelli, A., Batstone, D.J., Delgenès, J.P., Steyer, J.P., Ferrer, I., 2010. Pretreatment methods to improve sludge anaerobic degradability: A review. Journal of Hazardous Materials 183, 1-15.

386 Citulski, J.A., Farahbakhsh, K., 2010. Fate of Endocrine-Active Compounds during Municipal Biosolids Treatment: A Review. Environmental Science \& Technology 44, 83678376. 
Cobbledick, J., Aubry, N., Zhang, V., Rollings-Scattergood, S., Latulippe, D.R., 2016. Labscale demonstration of recuperative thickening technology for enhanced biogas production and dewaterability in anaerobic digestion processes. Water Research 95, 39-47.

Dhar, B.R., Nakhla, G., Ray, M.B., 2012. Techno-economic evaluation of ultrasound and thermal pretreatments for enhanced anaerobic digestion of municipal waste activated sludge. Waste Management 32, 542-549.

Greer, D., 2011. Municipal and industry synergies boost biogas production. BioCycle 52, 43. Habiba, L., Hassib, B., Moktar, H., 2009. Improvement of activated sludge stabilisation and filterability during anaerobic digestion by fruit and vegetable waste addition. Bioresource Technology 100, 1555-1560.

Hamid, H., Eskicioglu, C., 2013. Effect of microwave hydrolysis on transformation of steroidal hormones during anaerobic digestion of municipal sludge cake. Water Research 47, 4966-4977.

Kepp, U., Machenbach, I., Weisz, N., Solheim, O.E., 2000. Enhanced stabilisation of sewage sludge through thermal hydrolysis - three years of experience with full scale plant. Water Science and Technology 42, 89-96.

Kim, J., Park, C., Kim, T.-H., Lee, M., Kim, S., Kim, S.-W., Lee, J., 2003. Effects of various pretreatments for enhanced anaerobic digestion with waste activated sludge. Journal of Bioscience and Bioengineering 95, 271-275.

Liao, X., Li, H., Zhang, Y., Liu, C., Chen, Q., 2016. Accelerated high-solids anaerobic digestion of sewage sludge using low-temperature thermal pretreatment. International Biodeterioration and Biodegradation 106, 141-149.

Luo, Y., Guo, W., Ngo, H.H., Nghiem, L.D., Hai, F.I., Zhang, J., Liang, S., Wang, X.C., 2014. A review on the occurrence of micropollutants in the aquatic environment and their 
fate and removal during wastewater treatment. Science of The Total Environment 473-474, 619-641.

Malmborg, J., Magnér, J., 2015. Pharmaceutical residues in sewage sludge: Effect of sanitization and anaerobic digestion. Journal of Environmental Management 153, 1-10.

McNamara, P.J., Wilson, C.A., Wogen, M.T., Murthy, S.N., Novak, J.T., Novak, P.J., 2012.

The effect of thermal hydrolysis pretreatment on the anaerobic degradation of nonylphenol and short-chain nonylphenol ethoxylates in digested biosolids. Water Research 46, 29372946.

Mottet, A., Steyer, J.P., Déléris, S., Vedrenne, F., Chauzy, J., Carrère, H., 2009. Kinetics of thermophilic batch anaerobic digestion of thermal hydrolysed waste activated sludge. Biochemical Engineering Journal 46, 169-175.

Narumiya, M., Nakada, N., Yamashita, N., Tanaka, H., 2013. Phase distribution and removal of pharmaceuticals and personal care products during anaerobic sludge digestion. Journal of Hazardous Materials 260, 305-312.

Nghiem, L.D., Koch, K., Bolzonella, D., Drewes, J.E., 2017. Full scale co-digestion of wastewater sludge and food waste: Bottlenecks and possibilities. Renewable and Sustainable Energy Reviews 72, 354-362.

430 Nghiem, L.D., Manassa, P., Dawson, M., Fitzgerald, S.K., 2014. Oxidation reduction 431 potential as a parameter to regulate micro-oxygen injection into anaerobic digester for reducing hydrogen sulphide concentration in biogas. Bioresource Technology 173, 443-447. 
Phothilangka, P., Schoen, M.A., Huber, M., Luchetta, P., Winkler, T., Wett, B., 2008.

Prediction of thermal hydrolysis pretreatment on anaerobic digestion of waste activated sludge. Water Science \& Technology 58, 1467-1473.

Pilli, S., Yan, S., Tyagi, R.D., Surampalli, R.Y., 2015. Thermal Pretreatment of Sewage

Sludge to Enhance Anaerobic Digestion: A Review. Critical Reviews in Environmental Science and Technology 45, 669-702.

Reynolds, D.T., Cannon, M., Pelton, T., 2001. Preliminary investigation of recuperative thickening for anaerobic digestion. Proceedings of the Water Environment Federation 2001, $389-410$.

Sawatdeenarunat, C., Nguyen, D., Surendra, K.C., Shrestha, S., Rajendran, K., Khanal, S.K.,

Oechsner, H., Xie, L., 2016. Anaerobic biorefinery: Current status, challenges, and opportunities. Bioresource Technology.

Schieder, D., Schneider, R., Bischof, F., 2000. Thermal hydrolysis (TDH) as a pretreatment method for the digestion of organic waste. Water Science and Technology 41, 181-187. organic contaminants in biosolids: Impact of conventional wastewater and sludge processing technologies and emerging alternatives. Journal of Hazardous Materials 300, 1-17.

Semblante, G.U., Hai, F.I., Ngo, H.H., Guo, W., You, S.-J., Price, W.E., Nghiem, L.D., 2014.

Sludge cycling between aerobic, anoxic and anaerobic regimes to reduce sludge production during wastewater treatment: Performance, mechanisms, and implications. Bioresource Technology 155, 395-409. 
458 Sihuang, X., Hai, F.I., Xinmin, Z., Wenshan, G., Ngo, H.H., Price, W.E., Nghiem, L.D., 2016.

459 Anaerobic co-digestion: a critical review of mathematical modelling for performance 460 optimization. Bioresource Technology 222, 498-512.

461 Tadkaew, N., Hai, F.I., McDonald, J.A., Khan, S.J., Nghiem, L.D., 2011. Removal of trace 462 organics by MBR treatment: The role of molecular properties. Water Research 45, 2439-2451.

463 Torpey, N.W., Melbinger, R.N., 1967. Reduction of Digested Sludge Volume by Controlled 464 Recirculation. Journal Water Pollution Control 39, 1464-1474.

Tuyet, N.T., Dan, N.P., Vu, N.C., Trung, N.L.H., Thanh, B.X., De Wever, H., Goemans, M., Diels, L., 2016. Laboratory-scale membrane up-concentration and co-anaerobic digestion for energy recovery from sewage and kitchen waste. Water Science and Technology 73, 597-606.

Valo, A., Carrère, H., Delgenès, J.P., 2004. Thermal, chemical and thermo-chemical pretreatment of waste activated sludge for anaerobic digestion. Journal of Chemical Technology \& Biotechnology 79, 1197-1203.

Wijekoon, K.C., McDonald, J.A., Khan, S.J., Hai, F.I., Price, W.E., Nghiem, L.D., 2015.

Development of a predictive framework to assess the removal of trace organic chemicals by anaerobic membrane bioreactor. Bioresource Technology 189, 391-398. of trace organic contaminants in wastewater sludge and their removals by anaerobic digestion.

476 Bioresource Technology 210, 153-159.

477 Yang, S., McDonald, J., Hai, F.I., Price, W.E., Khan, S.J., Nghiem, L.D., 2017. The fate of trace organic contaminants in sewage sludge during recuperative thickening anaerobic digestion. Bioresource Technology. 
480 Yang, S., Nghiem, D.L., Bustamante, H., Rys, v.D., Murthy, N.S., 2015. Recuperative

481 thickening: A possible tool to improve anaeorbic digesiton of wastewater sludge. Proceedings 482 of the Ozwater 2015, Adelaide, Australia.

483 